\title{
Is Exercise-Induced Bronchoconstriction Exercise-Induced Asthma?
}

The article in this issue of Respiratory CARE by Burnett et $\mathrm{al}^{1}$ reports that 34 of 80 college athletes had exerciseinduced bronchoconstriction (EIB), most in the absence of symptoms. The authors further suggest that these athletes are at risk and that the identification of those with EIB may prevent morbidity and mortality. The implication is that the presence of EIB is evidence for asthma. But is the presence of EIB in the absence of exercise-induced dyspnea or other symptoms of asthma the same as exercise-induced asthma?

EIB in the normal population and in athletes has been described previously. ${ }^{2}$ The absence of apparent dyspnea associated with EIB was also seen when EIB was observed in children with rhinitis but no asthma. Others have reported as high as $40 \%$ prevalence of EIB associated with only allergic rhinitis. ${ }^{3}$ EIB was also reported in children with decreased levels of physical activity and no clinical evidence of asthma. ${ }^{4}$

But is EIB of clinical importance in the absence of dyspnea? Is a $>10 \%$ decrease in $\mathrm{FEV}_{1}$ subsequent to exercise in the absence of clinical dyspnea sufficient for the diagnosis of exercise-induced asthma? The presence of EIB in a child without symptoms of asthma may be, as suggested by Burnett et al, ${ }^{1}$ predictive that some minority will eventually develop symptoms of asthma, but most apparently will not. This study as well as most previous studies relating to EIB and asthma symptoms are cross-sectional. The higher incidence of EIB compared with symptoms in previous studies calls for longitudinal studies to determine a better or possibly more stringent criteria for EIB that might include higher cutoff value in $\mathrm{FEV}_{1}$ change, reproducibility of symptoms during testing, and reversibility with bronchodilators to help identify subjects at true risk of exercise-induced asthma and in need of attention and/or treatment.

We have previously reported ${ }^{5}$ our experience with exercise studies in 140 children examined because of a complaint of dyspnea on exertion. Most had been previously diagnosed and/or treated as having asthma by their primary care physician, but their negative response to albuterol before exercise was inconsistent with an asthma diagnosis. Despite reproducing their symptom of dyspnea on exertion, only 11 of those 140 had EIB. None of the others, most of whom were high school athletes, had EIB or other

The authors have disclosed no conflicts of interest.

Correspondence: Miles Weinberger MD. E-mail: miles-weinberger@uiowa.edu.

DOI: $10.4187 /$ respcare.04767 evidence of asthma. Their reproduced dyspnea on exertion was associated with various physiological findings. The difference in prevalence of EIB between our study ${ }^{5}$ and other

See the Original Study on Page 571

reports of EIB in subjects without asthma, including that of Burnett et al, ${ }^{1}$ is probably related to the more stringent criteria we used to define positive EIB. Our study included a higher cutoff value (drop in $\mathrm{FEV}_{1}$ of $>15 \%$ as opposed to $>10 \%$ ) than the study published in this issue of the Journal. Our study found EIB to be uncommon (8\%) in children seen because of dyspnea on exertion, whereas Burnett et $\mathrm{al}^{1}$ reported an EIB prevalence of $43 \%$ in college athletes, most having no respiratory symptoms. This raises the question of the clinical relevance for a modest degree of EIB in the absence of dyspnea on exertion or other symptoms of asthma.

\author{
Miles Weinberger MD \\ Emeritus Professor of Pediatrics \\ University of Iowa \\ Iowa City, Iowa \\ Mutasim Abu-Hasan MD \\ Department of Pediatrics \\ University of Florida \\ Gainesville, Florida
}

\section{REFERENCES}

1. Burnett DM, Burns S, Merritt S, Wick J, Sharpe MR. Prevalence of exercise-induced bronchoconstriction measured by standardized testing in healthy college athletes. Respir Care 2016;61(5):571-576.

2. Randolph C. An update on exercise-induced bronchoconstriction with and without asthma. Curr Allergy Asthma Rep 2009;9(6):433-438.

3. Carlsen KH, Anderson SD, Bjermer L, Bonini S, Brusasco V, Canonica W, et al. Exercise-induced asthma, respiratory and allergic disorders in elite athletes: epidemiology, mechanisms and diagnosis: part I of the report from the Joint Task Force of the European Respiratory Society (ERS) and the European Academy of Allergy and Clinical Immunology (EAACI) in cooperation with GA2LEN. Allergy 2008;63(4):387-403.

4. Anthracopoulos MB, Fouzas S, Papadopoulos M, Antonogeorgos G, Papadimitriou A, Panagiotakos DB, et al. Physical activity and exercise-induced bronchoconstriction in Greek schoolchildren. Pediatr Pulmonol 2012;47(11):1080-1087.

5. Abu-Hasan M, Tannous B, Weinberger M. Exercise-induced dyspnea in children and adolescents: if not asthma then what? Ann Allergy Asthma Immunol 2005;94(3):366-371. 